\title{
Paget's disease of male breast, a rare but rising ailment
}

\author{
Randhir Sagar Yadav, ${ }_{1}^{1}$ Shumneva Shrestha, ${ }^{1}$ Prabesh Bikram Singh, ${ }^{1}$ Sadikshya Sharma, ${ }^{2}$ Bikash Nepal, ${ }^{3}$ Prakash \\ Sayami $^{4}$
}

${ }^{1}$ Maharajgunj Medical Campus, Institute of Medicine, Kathmandu,

${ }^{2}$ College of Medical Sciences, Bharatpur,

${ }^{3}$ Department of Surgery, Tribhuvan University Teaching Hospital, Kathmandu,

${ }^{4}$ Manmohan Cardio-thoracic Vascular and Transplant Center, Institute of Medicine, Tribhuvan University, Kathmandu

Correspondence: Randhir Sagar Yadav,

Email: sagar.randhir@gmail.com

\begin{abstract}
w
Male breast cancer constitutes less than $1 \%$ of all breast carcinomas whereas Paget's disease accounts for less than $1 \%$ of entire breast cancers. Unawareness and misdiagnosis lead to delayed presentation at a higher grade making the prognosis worse than females. Genetics and hormonal factors are strongly linked to its occurrence. Investigations, diagnosis, staging and management strategies for male breast cancers are concluded from studies done in female cases. We present a case of a 65 years literate gentleman who presented with fungating right breast mass with bleeding ulcer who underwent modified radical mastectomy, chemotherapy, radiotherapy and hormonal therapy.
\end{abstract}

Key words: Male breast cancer; Paget's disease; Prognosis.

\section{Introduction}

Breast cancer in male is a rare occurrence, which comprises less than $1 \%$ of all breast cancers. ${ }^{1}$ It usually presents as a subareolar mass in elderly where the density of breast tissue is maximum. Paget's disease (PD) of the nipple is the superficial manifestation of the underlying breast carcinoma. It accounts for less than $1 \%$ of all breast cancers. ${ }^{2}$ Thus, $\mathrm{PD}$ of nipple in male is an extremely rare ailment. Invasive ductal carcinoma remains the most common histologic. ${ }^{3}$ In USA, the incidence of male breast cancer has raised from 0.86 to 1.08 per 10,000 men. $^{3}$ However, the incidence for Nepal has not been reported.

\section{Case report}

A 65 years male with university degree presented to the surgical OPD of Tribhuvan University Teaching Hospital with a fungating right breast mass for 5 months. It was associated with pricking pain and bloody discharge. The patient appreciated the growing mass as the pain progressed. He received several courses of antibiotics and non-steroidal anti-inflammatory drugs. He had been on medications for diabetes mellitus and hypertension for past three decades. He was a nonsmoker and didn't consume alcohol. There was no family history of any cancer.

On physical examination, there was $3 \mathrm{~cm}$ X $3 \mathrm{~cm}$ fungating mass with active bleeding (Figure 1). It was irregular, firm and tender. Axillary lymph nodes were palpable and matted. Supraclavicular lymph node was not palpable. Biopsy reveled grade II invasive carcinoma of no special type. Lymphatic invasion was seen whereas vascular and perineural invasions were not identified. Contrast Enhanced Computed Tomography (CECT) of chest reported features suggestive of breast carcinoma with invasion of pectoral muscle and erosion of $4^{\text {th }}$ rib. Axillary lymph nodes were enlarged which encased axillary vessel leading to luminal narrowing with features of necrotic changes (Figure 2). 


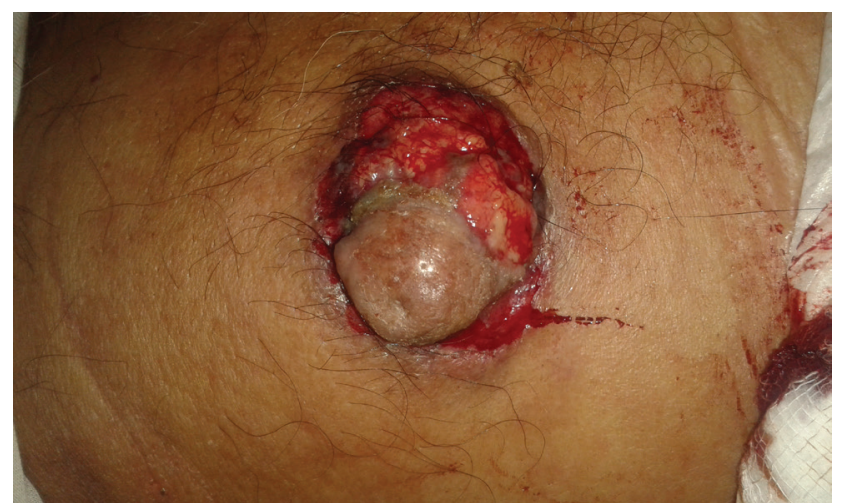

Figure 1: A fungating right breast mass

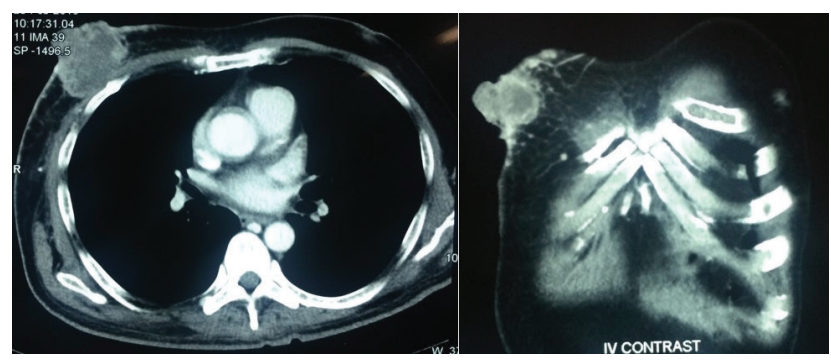

Figure 2: CECT of chest suggestive of breast carcinoma with erosion of $4^{\text {th }}$ rib

He received three cycles of neoadjuvant chemotherapy (Docetaxel, Epirubucin and Cyclophosphamide). There was considerable downsizing of the tumor mass with cessation of bleeding. Chest X-ray and USG abdomen showed no metastasis. Preoperative investigations were within normal limits. Then Modified Radical Mastectomy (MRM) was done. Peroperatively, approximately $3 \mathrm{~cm} \mathrm{X}$ $2 \mathrm{~cm}$ fungating right nipple mass was resected. On axillary dissection, lymph nodes were densely adherent to the axially vein. Six axillary lymph nodes were resected. Postoperative period was uneventful. Postoperative histopathology revealed grade II invasive carcinoma of no special type. Lymphovascular invasion was seen whereas perineural invasion was not seen. All resected margins were free however, 5 among 6 lymph nodes showed metastasis. (Figure 3). Nipple-areola was diagnosed as PD. AJCC (TNM) stage was pT1cN2. Immunohistochemistry findings showed Estrogen Receptor (ER) status positive while Progesterone receptor (PR) status and Her-2/ Neu were negative. Post-MRM, he received three cycles of adjuvant chemotherapy. Radiotherapy and hormonal therapy (Tamoxifen $20 \mathrm{mg}$ /day) were subsequently given. On follow up the patient has no recurrence.

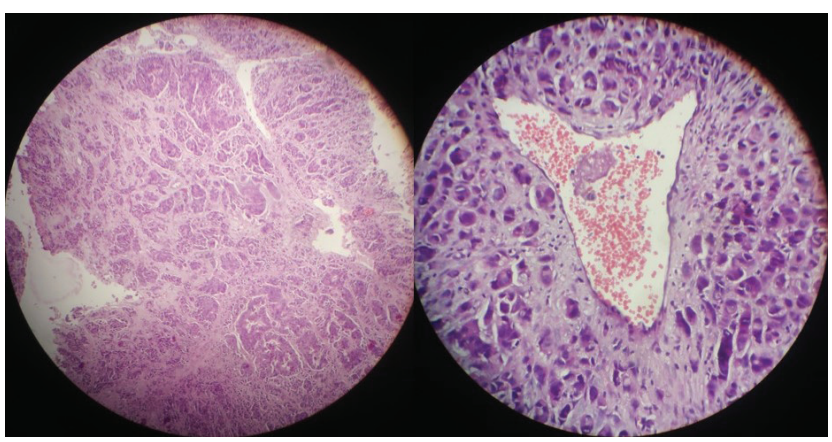

Figure 3: Histopathological finding of invasive carcinoma of no special type and vascular invasion

\section{Discussion}

Despite the rarity, there has been a notable increase in the incidence of male breast carcinoma. ${ }^{3}$ Data on the incidence and the recent trend in Nepalese patients is hardly available. There are biological similarities in both male and female breast cancers with some basic pathological differences. ${ }^{4}$ Unlike female breast cancer studies, much about the epidemiology of male equivalent is yet to be known. In contrast to the bimodal age of distribution in females, male cases have single peak at elderly age. ${ }^{4}$ They present about 5 to 10 years later than females in USA whereas there is a smaller range difference in the rest of countries. ${ }^{5}$ A range of 1-8 months duration between occurrence of symptoms and presentation at health facilities has been noted. ${ }^{6}$ The median age at diagnosis of male breast cancer is 67 years. ${ }^{1}$ These were somewhat consistent to our patient who was 65 years and presented after 5 months. There are several risk factors for the development of male breast carcinoma. BRCA2 mutations more than BRCA1 mutations are associated with male breast cancers. Similarly, family history, gynaecomastia, radiation, testicular diseases and hormonal therapy are other risk factors. ${ }^{1}$ Klinefelter syndrome and Cowden disease are the associated genetic diseases. ${ }^{7}$ There was no such known risk factor in this case. Studies have shown estrogen and progesterone receptors present in about $90 \%$ and $81 \%$ of male breast cancers respectively. ${ }^{3}$ In males, it usually present with painless subareolar lump, nipple retraction, discharge and ulceration. ${ }^{3,6}$ Occurrence of nipple and lymph node involvement as well as lymphovacular invasion is more common in male breast cancers than females. ${ }^{8}$ Our case had a painful and tender mass with bloody discharge involving nipple. Tumor size and status of lymph node are two prominent determinant of outcome in male cases. ${ }^{3}$ There were matted palpable axillary lymph nodes in our patient. Most of the initial male breast lumps misrepresent as gynaecomastia. This misdiagnosis along with delay in seeking care by patients lead to late 
diagnosis. $^{9}$ Our patient also remained in contact with local paramedicals who treated this mass as an infective wound. Early diagnosed cases are usually found at low or intermediate grade. As high as $42 \%$ male breast carcinomas are diagnosed at stages III or IV. ${ }^{7}$ Our case was diagnosed at stage III. As they are diagnosed at higher stages, male breast carcinomas show a poorer overall prognosis than females. ${ }^{3}$ On contrary, a comparative study adjusted for stage and age showed males to have a comparable or better prognosis than females. ${ }^{1}$ Data on applicable diagnostic modality in male breast abnormalities are scarcely available. Similar in females, judicious triple assessment remains the mainstay of the diagnosis of male breast cancers. ${ }^{1}$ Tissue biopsy provides definitive diagnosis with evidences on estrogen and progesterone receptors as well as Her-2/ Neu status. ${ }^{10}$ Clinically at presentation there was a fungating mass with bleeding ulcer quite impressive of locally advanced disease. Patient was subjected to skin edge biopsy which established diagnosis and thus mammography was not considered. Like the diagnostic modalities, treatment approaches for male breast cancers are inferred from studies done in female breast carcinomas. ${ }^{1,4}$ Upfront surgery and upfront chemotherapy is done for early stage and locally advanced breast cancer respectively. In clinically axillary lymph node negative cases, simple mastectomy or MRM with sentinel node biopsy is performed. Similarly, axillary clearance is done in clinically node positive cases. ${ }^{6}$ Our patient was opted for three cycles of upfront chemotherapy which showed partial response. Finally MRM was done. Likewise, post mastectomy criteria for radiation in male is again deduced from female data. ${ }^{11}$ Usually male having tumor size $>5 \mathrm{~cm}$ or involvement of 4 or more lymph nodes necessitates the use of post-surgery radiation. Our patient showed metastasis in 5 out of 6 lymph nodes. Post-MRM adjuvant chemotherapy and radiotherapy was given. As seen in female, stage is regarded a significant prognostic factor in male breast cancers. The relative risk of breast cancer deaths is 1.5 fold higher in male cases than females. ${ }^{12}$ Amidst available limited data on male breast cancers, further studies are needed to compare prognosis in both sexes. There is 93 times increased risk of developing a contralateral primary breast cancer in males. ${ }^{1}$ Male breast cancer survivors do possess significant risk of developing prostatic cancer. Stomach cancer and cutaneous melanoma may occur as second cancers. ${ }^{13}$ This warrants timely and appropriate screenings in them. Our patient is in close follow up with a team of oncologists.

\section{Conclusion}

High degree of suspicion in male patients with breast lump is of outmost importance to improve the management and prognosis. All male breast lumps should be evaluated to rule out carcinoma. Neoadjuvant therapy and radical surgery offers best outcome.

\section{References}

1. Giordano SH. A Review of the Diagnosis and Management of Male Breast Cancer. Oncologist. 2005;10(7):471-9. https://doi.org/10.1634/theoncologist.10-7-471

2. ElHarroudiT,TijamiF,ElOtmanyA,JalilA.Pagetdisease of the male nipple. J Cancer Res Ther. 2010;6:95-6. https://doi.org/10.4103/0973-1482.63561

3. Giordano SH, Cohen DS, Buzdar AU, Perkins G, Hortobagyi GN: Breast carcinoma in men: a population-based study. Cancer. 2004;101:51-57. https://doi.org/10.1002/cncr.20312

4. Giordano SH, Buzdar AU, Hortobagyi GN: Breast cancer in men. Ann Intern Med. 2002;137:678-687. https://doi.org/10.7326/0003-4819-137-8-20021015000013

5. Salehi A, Zeraati H, Mohammad K et al. Survival of male breast cancer in Fars, South of Iran. Iran Red Crescent Med J. 2011;13(2):99-105.

6. Agrawal, A., Ayantunde, A. A., Rampaul, R. \& Robertson, J. F. Male breast cancer: a review of clinical management. Breast Cancer Res Treat. 2007;103(1):11-21. https://doi.org/10.1007/s10549-006-9356-z

7. Fentiman IS, Fourquet A, Hortobagyi GN: Male breast cancer. Lancet. 2006;367:595-604. https://doi.org/10.1016/S0140-6736(06)68226-3

8. McLachlan SA, Erlichman C, Liu FF et al. Male breast cancer: an 11 year review of 66 patients. Breast Cancer Res Treat. 1996;40(3):225-230. https://doi.org/10.1007/BF01806810

9. Thalib L, Hall P. Survival of male breast cancer patients: population-based cohort study. Cancer Sci. 2009;100(2):292-295. https://doi.org/10.1111/j.1349-7006.2008.01032.x

10. Jemal A, Siegel R, Ward E, Hao Y, Xu J, Thun MJ: Cancer statistics. CA Cancer J Clin. 2009;59:225-249. https://doi.org/10.3322/caac.20006 
JSSN 2017; 20 (2)

11. Chakravarthy A, Kim CR. Postmastectomy radiation in male breast cancer. Radiother Oncol. 2002;65(2):99-103. https://doi.org/10.1016/S0167-8140(02)00210-4

12. Herman K, Lobaziewicz W, Skotnicki P et al. Male breast cancer. Does the prognosis differ compared to female? Neoplasma. 2000;47(3):191-195.

13. Lee UJ, Jones JS. Incidence of prostate cancer in male breast cancer patients: a risk factor for prostate cancer screening. Prostate Cancer Prostatic Dis. 2009;12(1):52-56. https://doi.org/10.1038/pcan.2008.26 